Dynamics of laminar triple-flamelet structures in non-premixed turbulent combustion

Dold, J W and Hartley, L J and Green, D

1991

MIMS EPrint: 2007.89

Manchester Institute for Mathematical Sciences

School of Mathematics

The University of Manchester

\footnotetext{
Reports available from: http://eprints.maths.manchester.ac.uk/

And by contacting: The MIMS Secretary

School of Mathematics

The University of Manchester

Manchester, M13 9PL, UK
} 


\title{
DYNAMICS OF LAMINAR TRIPLE-FLAMELET STRUCTURES IN NON-PREMIXED TURBULENT COMBUSTION*
}

\author{
J.W. DOLD, L.J. HARTLEY, AND D. GREEN†
}

\begin{abstract}
In the spirit of laminar-flamelet modelling of non-premixed turbulent combustion, a diffusion flamelet is studied. However, the flamelet is also taken to end at a finite position. Such an end of a diffusion flame exhibits fuel-rich and fuel-lean premixed elements as well as the diffusion flame-sheet itself - a structure that is known as a triple-flame and which has the property of being able to propagate. A counterflow geometry with shear becomes the most relevant situation in which to picture ends of diffusion flames in a turbulent flow. In an equidiffusive system, the speed of propagation of the end-point is demonstrated to be positive only for relatively limited values of the strain or scalar dissipation rate and becomes large and negative towards the higher finite value at which a diffusion flame would extinguish uniformly. The implications of these findings for the behaviour of turbulent diffusion flames are discussed.
\end{abstract}

\section{INTRODUCTION.}

This article is intended to summarise a number of ideas that show how properties of two-dimensional triple-flame structures can have implications for the dynamical role of propagating or receding flame sheets in non-uniform and non-premixed (possibly turbulent) combustion. A more detailed discussion appears elsewhere [1].

The burning of a fuel and an oxidant under conditions in which they are initially separated is typically characterised by the existence of thin diffusion flames at which fuel and oxidant are converted into reaction products in stoichiometric proportions [2]. This simple picture reduces the description of such combustion to the analysis of only reactionless diffusive processes in which any diffusion flame appears in the form of a (possibly moving) boundary condition [3].

The thickness of the diffusion flame is governed, essentially, by the local Damköhler number of the chemistry, which measures the ratio of a characteristic time-scale for diffusion $t_{d}$ in the combustion setup to a characteristic time-scale for chemical changes $t_{c}$. The first noticeable result of reducing the Damköhler number is to broaden the flame structure [4]. In complex chemical schemes, involving many reaction steps, this can become very important with the time-scales for some individual reaction steps becoming so long that intermediate chemical products and some pollutants may be able to 'escape' from the otherwise thin flame. The flame becomes a source for non-equilibrium products, a fact that may have sometimes useful and sometimes unpleasant implications. Steady flames behaving in this way can be analysed asymptotically or numerically in a conceptually straightforward way, as for example in references [5]-[7].

The kind of mixing rates at which non-equilibrium effects become significant in combustible systems is probably achieved most readily in turbulent flows. A turbulent

*Prepared for 'Dynamical Issues in Combustion Theory,' P.C. Fife, A.A. Liñán and F.A. Williams (Eds.), IMA Volumes in Mathematics and its Applications, Springer Verlag.

†School of Mathematics, University of Bristol, Bristol BS8 1TW, United Kingdom. 
diffusion flame, however, is very difficult to analyse mathematically and numerically. Not only is a wide range of mixing rates generated by the turbulence, but these fluctuate rapidly and chaotically both in position and time. One approach that has met with some success is based on the hypothesis that the flame burns in the form of locally quasi-steady 'laminar-flamelets' at any moment in the evolution. Reviews of this type of approach applied to diffusion flames are available in references $[8,9]$.

Briefly, this allows one to use more straightforward steady analyses to calculate (possibly multiple) solutions for flame structures in any conceivable locally laminar flow consideration that might be produced by the turbulence. It is most natural to express such solutions in terms of a mixture fraction parameter $Z$, or any similar parameter that varies monotonically across any flame between (say) zero in the oxidising atmosphere and one at the source of fuel. This family of laminar flame solutions is then assumed to describe the instantaneous response of the chemistry to the turbulent flow at any moment, thus divorcing the detailed calculation of the chemistry from the calculation of the turbulence.

The most useful way of expressing the resulting interaction between flow and chemistry may then be posed in the form of a statistical description [10]. Accordingly, if $\boldsymbol{f}(Z, \boldsymbol{g})$ is a vector of chemical attributes $\boldsymbol{f}$ (such as temperature and concentrations) calculated as a function of $Z$ for the vector of local flow attributes $\boldsymbol{g} \in \mathcal{D}$ (such as strain-rate and vorticity) and if $P(Z, \boldsymbol{g} ; \boldsymbol{r}, t)$ is the joint probability density of encountering the flow properties $\boldsymbol{g}$ and mixture fraction $Z$ at any point $\boldsymbol{r}$ and time $t$, then the expected (or mean) values of $\boldsymbol{f}$ can be estimated by

$$
\widetilde{\boldsymbol{f}}(\boldsymbol{r}, t)=\iint \boldsymbol{f}(Z, \boldsymbol{g}) P(Z, \boldsymbol{g} ; \boldsymbol{r}, t) d \boldsymbol{g} d Z
$$

in which the integration is taken over the entire domain of $(Z, \boldsymbol{g})$, namely $[0,1] \times \mathcal{D}$.

This approach works moderately well in describing weakly turbulent diffusion flames $[8,9]$ where Damköhler numbers are sufficiently large to ensure a rapid transient response of the chemistry to any changes in the flow properties at any point, and where laminar flamelet solutions $f(Z, g)$ are unique. However, conceptual difficulties arise in those parts of the domain of $\boldsymbol{g}$ where nonlinear chemical effects can lead to multiple (or even potentially oscillatory) solutions $[5,11]$. Not only does the choice of solution in the expression (1) become a problem, but questions of stability and significant transient effects, as $\boldsymbol{g}$ crosses solution boundaries, have to be addressed. A simple one dimensional description of laminar flamelets $f(Z, g)$ ceases to be acceptable and dynamical aspects can no longer be ignored.

A typical way in which multiplicity can be encountered in diffusion flames may be thought of in terms of so-called 'ignition' and 'extinction' limits. These are found if the chemistry is modelled using a single step temperature-sensitive reaction [5] and would provide a qualitatively correct description for many more complicated chemical schemes. A sketch of the situation appears in figure 1. At one extreme, large values of $g \in \mathcal{D}_{E} \subset \mathcal{D}$ would give rise to Damköhler numbers that are too small for significant chemical change to be possible-only a cold or 'extinguished' solution can then describe the chemistry. However as $g$ is reduced across the boundary $\partial \mathcal{D}_{E}$, a turning point bifurcation is found to a second unstable intermediate range of solutions representing a regime of 'partially premixed' combustion [5]. 


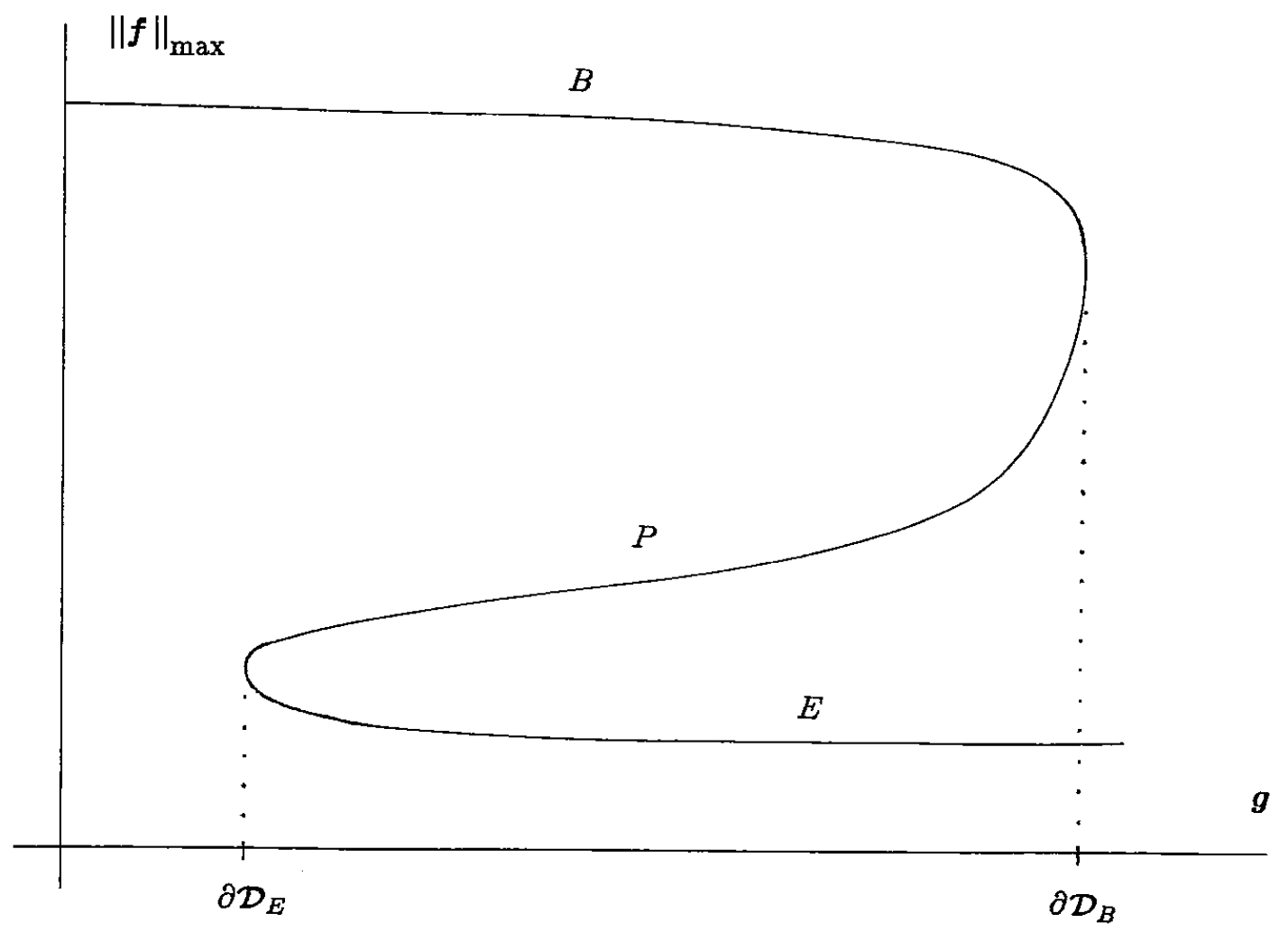

Figure 1. Typical multiple branches of solution, showing the domains of extinguished, $E$, and burning, $B$, solutions as well as the intermediate 'partially mixed' branch, $P$.

This boundary is usually referred to as an 'ignition limit' because any cold initial conditions in the domain $g \in \mathcal{D} \backslash \mathcal{D}_{E}$ would necessarily be unsteady and would evolve towards a hot 'burning' solution via some self-ignition or reaction runaway process [12]-[14]. It must be said, however, that the transient time-scale for this ignition process is typically very long. Under normal room-temperature conditions it may be of the order of decades, so that unless fuel and oxidant streams are initially very hot this one-dimensional reaction runaway transient is unlikely to be significant in real turbulent diffusion flames.

On the other hand, the hot diffusion flame solution is found to persist throughout a larger domain $g \in \mathcal{D}_{B} \supset \mathcal{D} \backslash \mathcal{D}_{E}$. In this case the boundary, $\partial \mathcal{D}_{B}$ marks a second turning point bifurcation to the intermediate branch of partially premixed solutions. The latter branch, and in fact all three types of solution, exist throughout the non-empty domain $\mathcal{D}_{P}=\mathcal{D}_{E} \cap \mathcal{D}_{B}$. Since only the cold solution can be found in $\mathcal{D} \backslash \mathcal{D}_{B}$, the boundary $\partial \mathcal{D}_{B}$ marks an 'extinction limit.' If $\boldsymbol{g}$ enters this range, any hot solution extinguishes quickly enough for this particular transition to play 
a potentially major role in turbulent non-premixed combustion. For example, its effects could range between causing some degree of incomplete burning and making the entire flame go out [15].

The most important implication of this extinction boundary is that the function $\boldsymbol{f}(Z, \boldsymbol{g})$ may sometimes need to be assigned cold or 'extinguished' values in equation (1) if there is any possibility, however remote, of values of $\boldsymbol{g}$ entering the extinction domain $\mathcal{D} \backslash \mathcal{D}_{B}$. Essentially, such solutions would represent 'holes' in diffusion flames $[3,16]$ the edges of which have the most noteworthy feature of marking boundaries between cold regions where fuel and oxidant can mix without reacting and regions where diffusion flames consume any reactants. Such boundaries typically involve fuel-rich and fuel-lean premixed flames as well as the diffusion flame itself.

Some studies have been made into these structures, which have become known as 'triple-flames' [16]-[25]. A photograph of such a flame is reproduced in figure 2. Unlike any relatively passive diffusion flame, such flame formations are able to propagate, which means that a dynamic behaviour must be considered in describing holes in diffusion flames or, in particular, their edges. As Williams points out [3, p. 409], this dynamic is clearly very important in ascertaining the role of such holes in turbulent non-premixed combustion.

The main purpose of this paper is to describe the way in which a two dimensional laminar triple-flamelet can be used to augment the simple one-dimensional flamelet $\boldsymbol{f}(Z, \boldsymbol{g})$. In particular, both positive and negative speeds of propagation of tripleflamelets can be encountered-a negative propagation speed meaning simply that an extinction front advances itself into the diffusion flame [25]. A simple model example is considered in order to reveal some of the essential underlying flame structures that lead to either positive or negative propagation. It should be noted that the model is open to the generalisation of including stronger thermal expansion effects, nonunit Lewis numbers (different diffusivities of heat and reactants) and more complex chemical schemes. This is a potential area of study that is still new and that is currently being actively investigated. At this stage, it is worth pointing out that surprising effects may be anticipated in varying, for example, the Lewis number [25][27].

Having demonstrated the existence of such triple-flamelet solutions using the simple model, their more general implications for the dynamic behaviour of holes in strained diffusion flames are considered. Some conclusions are reached about the way in which this information would need to be incorporated into any probabilistic model for describing turbulent diffusion flames.

\section{MODEL.}

2.1 Reactive-Diffusive System. If it is assumed that a single-step chemical reaction $F+X \rightarrow 2 P$ takes place to combine one fuel molecule $F$ with one oxidant molecule $X$ in producing the product $P$, then a suitable low Mach number model 


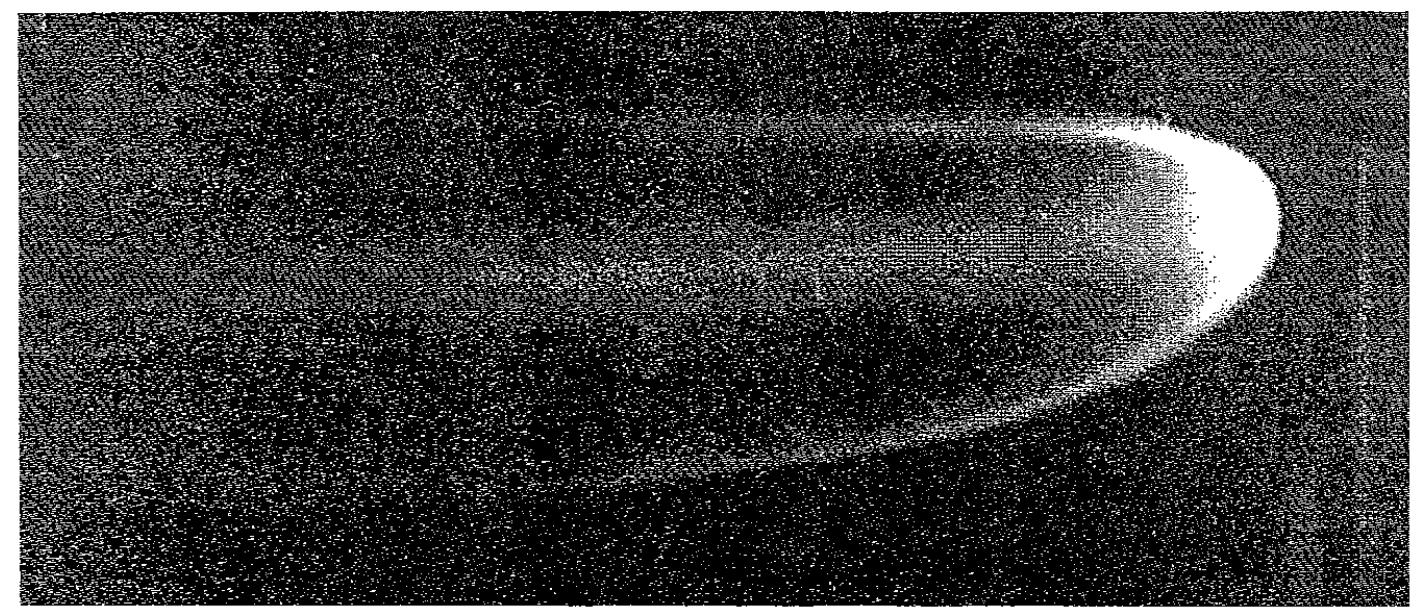

Figure 2. A triple flame propagating in a non-uniform mixture of methane and air, showing a fuel-rich premixed flame above, fuel-lean flame below, and a trailing diffusion flame-British Crown Copyright, reproduced from [17] by kind permission of $\mathrm{H}$. Phillips, Health and Safety Executive, Buxton, U.K.

for the evolution of fuel, oxidant and temperature is

$$
\begin{array}{rr}
\rho \dot{c}_{\alpha}-\nabla \cdot\left(\rho D_{\alpha} \nabla c_{\alpha}\right)=-W_{\alpha}\left(\rho c_{F}\right)\left(\rho c_{X}\right) k \\
\\
\text { where } & k(T)=\frac{1}{t_{0}} \exp \left(-T_{A} / T\right)
\end{array}
$$

in which the dot notation signifies a Lagrangian or particle-following differential operator (for example $\dot{c}_{F}=\partial c_{F} / \partial t+\boldsymbol{u} \cdot \nabla c_{F}$ ). Also, $W_{\alpha}$ denotes the molecular weight, $c_{\alpha}$ is the mass-fraction and $D_{\alpha}$ is the diffusion coefficient of the species $\alpha \in\{F, X\}$; the specific heat at constant pressure is $C_{P}$, thermal conductivity is $\lambda$ and $Q$ represents the molar heat of reaction. The constant $T_{A}$ is an Arrhenius activation temperature and $t_{0}^{-1}$ is a molecular frequency factor of the reaction. If the rate 'constant' $k(T)$ is to be a sensitive function of temperature, as it is in many realistic combustion reactions, then the activation temperature must be large, $T_{A} \gg T$, 
for all temperatures of interest.

In order to simplify the analysis as much as possible, the quantities $C_{P}, \lambda, \rho D_{\alpha}$ and $Q$ will all be treated as constant. Moreover, a constant density assumption will be invoked. For gaseous combustion, this is only strictly justifiable in the limit of small heat release, $Q \ll C_{P} T$, but it does considerably simplify the analysis by focussing attention mainly on the primary reactive, diffusive and convective effects. As such, the important qualitative features of the relevant diffusion and triple flames (or flamelets) are retained without the added complexity of velocity and chemical interactions. For the purpose of modelling these flames, a divergence free velocity, $\boldsymbol{\nabla} \cdot \boldsymbol{u}=0$, will simply be specified. The model can be extended to include density and pressure changes, with an analogous treatment to that presented below, simply by adding a suitable equation of state and a momentum equation.

A 'conserved scalar' is any linear combination of quantities, such as atoms or total energy, that are neither created nor destroyed by chemical changes. As a result their consideration can provide some additional simplification of the model. For this purpose, the reaction-rate terms in equations (2) can be eliminated so as to identify a mixture fraction $Z$ and specific enthalpies $H_{F}$ and $H_{X}$ associated with the fuel and oxidant streams, respectively, as follows:

$$
\begin{gathered}
Z-S=\left(\frac{c_{F}}{W_{F}}-\frac{c_{X}}{W_{X}}\right) /\left(\frac{c_{F 0}}{W_{F}}+\frac{c_{X 0}}{W_{X}}\right) \quad: \quad S=\frac{c_{X 0}}{W_{X}} /\left(\frac{c_{F 0}}{W_{F}}+\frac{c_{X 0}}{W_{X}}\right), \\
H_{F}=Q \frac{c_{F}}{W_{F}}+C_{P}\left(T-T_{X 0}\right) \quad \text { and } \quad H_{X}=Q \frac{c_{X}}{W_{X}}+C_{P}\left(T-T_{F 0}\right) .
\end{gathered}
$$

With $c_{F}$ and $c_{X}$ having the respective values $c_{F 0}$ and $c_{X 0}$ in their own originally separate streams, and zero in the alternative streams, the mixture fraction $Z$ is thus defined to vary between 0 and 1 . The mixture is stoichiometric, containing one fuel molecule to every oxidant molecule, at the value $Z=S$. The temperatures in the original fuel and oxidant streams are taken to be $T_{F 0}$ and $T_{X 0}$, respectively.

Because $Z, H_{F}$ and $H_{X}$ are not linearly independent, it becomes convenient to pose the problem in terms of (say) $Z, H_{F}$ and $T$. The model equations then take the form

$$
\begin{aligned}
& \dot{Z}-D_{X} \nabla^{2} Z=\frac{\left(D_{X}-D_{F}\right) / Q}{c_{F 0} / W_{F}+c_{X 0} / W_{X}} \nabla^{2}\left(C_{P} T-H_{F}\right) \\
& \dot{H}_{F}-D_{F} \nabla^{2} H_{F}=\left(\kappa-D_{F}\right) \nabla^{2}\left(C_{P} T\right) \\
& \dot{T}-\kappa \nabla^{2} T=\frac{\rho W_{F} W_{X}}{C_{P} Q} k(T)\left\{H_{F}-C_{P}\left(T-T_{X 0}\right)\right\} \times \\
& \times\left\{H_{F}-C_{P}\left(T-T_{X 0}\right)+Q(S-Z)\left(\frac{c_{F 0}}{W_{F}}+\frac{c_{X 0}}{W_{X}}\right)\right\}
\end{aligned}
$$

in which $\kappa$ represents the thermal diffusivity, $\kappa=\lambda /\left(\rho C_{P}\right)$. These equations make it clear that non-chemical source terms can still exist for the conserved scalars $Z$, $H_{F}$ and $H_{X}$ if the diffusivities $D_{F}, D_{X}$ and $\kappa$ are not all equal.

Equidiffusive Adiabatic Model: For simplicity and for illustrative purposes, we will not consider this more general case here [25], and instead restrict attention to 
cases in which mass and heat are equidiffusive, $D_{F}=D_{X}=\kappa$. Only the fundamental chemical source term in the temperature equation then survives.

The equations for $Z$ and $H_{F}$ are then identical. Over most of a turbulent flow, the initial and boundary conditions for $Z$ and $H_{F}$ may also be considered to be similarthis may require assuming either 'distant' or insulating impermeable boundaries at which $Z$ and $H_{F}$ would both satisfy Neumann conditions. At any rate, it becomes reasonable to expect that $H_{F}$ and $Z$ would become linearly related, so that

$$
H_{F}=\left[Q \frac{c_{F 0}}{W_{F}}+C_{P}\left(T_{F 0}-T_{X 0}\right)\right] Z
$$

Taking this adiabatic relation to hold, and also taking the temperatures $T_{X 0}$ and $T_{F 0}$ to both equal $T_{0}$, the model can now be redrafted into a straightforward and considerably simplified form.

Since $c_{F}$ and $c_{X}$ are non-negative, the temperature must be bounded above by $T_{S}=T_{0}+Q S c_{F 0} /\left(W_{F} C_{P}\right)$. Defining a dimensionless temperature $\tilde{T}$ that varies between 0 and 1 , and referring time, space and velocity scales to $t_{c}, r_{c}$ and $u_{c}$, such that

$$
\begin{array}{ccc}
T= & T_{0}+\left(T_{S}-T_{0}\right) \tilde{T} & t_{c}=\frac{\beta^{4}}{\rho\left(c_{F 0} W_{X}+c_{X 0} W_{F}\right) k\left(T_{S}\right)} \\
r_{c}=\left(\kappa t_{c}\right)^{1 / 2} & \text { and } & u_{c}=r_{c} / t_{c}
\end{array}
$$

the model equations become

$$
\begin{gathered}
Z_{t}+\tilde{\boldsymbol{u}} \cdot \tilde{\nabla} Z-\tilde{\nabla}^{2} Z=0 \\
\tilde{T}_{t}+\tilde{\boldsymbol{u}} \cdot \tilde{\nabla} \tilde{T}-\tilde{\nabla}^{2} \tilde{T}=\beta^{4}[Z-S \tilde{T}][1-Z-(1-S) \tilde{T}] \exp \left(\frac{\beta(\tilde{T}-1)}{1+\alpha(\tilde{T}-1)}\right)
\end{gathered}
$$

where

$$
\beta=\alpha T_{A} / T_{S} \quad \text { with } \quad \alpha=\left(T_{S}-T_{0}\right) / T_{S} .
$$

Because $T_{A} \gg T_{S}$, the 'Zeldovich number' $\beta$ can be considered to be large, $\beta \gg 1$. This provides an extremely useful key to analysing these model equations asymptotically [5]. At the stoichiometric value $S$ of $Z$, the temperature $\tilde{T}$ reaches its strict upper bound of unity if and only if all fuel and oxidant are consumed by the reaction-both square brackets in the equation for $\tilde{T}$ then being zero.

2.2 Triple-Flamelet Model. Let us now consider the moving surface in space $\{Z \equiv S\}$ defined as $\{\boldsymbol{r} \mid Z(\boldsymbol{r}, t) \equiv S\}$. Since the exponential reaction-rate term in equations (7) is significant (for $\beta \gg 1$ ) only if $1-\tilde{T}$ is small, any quasi-steady diffusion flame must lie in a small neighbourhood about this surface, with $\tilde{T}>\tilde{T}_{c}=$ $1-O\left(\beta^{-1}\right)$ on $\{Z \equiv S\}$. The constant $\tilde{T}_{c}(\beta, S)$ may be thought of as any temperature just below the critical temperature for extinction of a uniform fully-steady diffusion flame [5], or any convenient lower temperature. Defining another moving surface $\left\{\tilde{T} \equiv \tilde{T}_{c}\right\}$ as $\left\{\boldsymbol{r} \mid \tilde{T}(\boldsymbol{r}, t) \equiv \tilde{T}_{c}\right\}$, any non-empty intersection $\{Z \equiv S\} \cap\left\{\tilde{T} \equiv \tilde{T}_{c}\right\}$ would then represent at least one path in $\{Z \equiv S\}$ across which the diffusion flame must come to an end. Equivalently, it would represent the union of all of the edges of all of the holes in all of the diffusion flames. 
In order to examine the local behaviour of any point on the edge of one of these holes, it is natural to select orthogonal moving coordinates, (say) $y$ normal to $\{Z \equiv$ $S$ \}, with $y$ increasing as $Z$ increases, $z$ tangent to $\{Z \equiv S\} \cap\left\{\tilde{T} \equiv \tilde{T}_{c}\right\}$, and $x$ increasing into the diffusion flame. The axes of $x, y$ and $z$ would generally rotate as well as translate as they follow the movement of the surface $\{Z \equiv S\}$ and the edge of the hole.

A laminar triple-flamelet model can then be constructed by invoking a locally quasi-steady slowly-varying approximation. Accordingly, one would expect to find that solutions take on the functional forms, $Z=Z(y)$ and $\tilde{T}=\tilde{T}(x, y)$, after neglecting any transients and any nonlocal behaviour (including curvature effects) at large values of $x, y, z$ and $t$. This amounts to having predominantly low-frequency components [8] in the turbulent velocity field $\boldsymbol{u}(\boldsymbol{r}, t)$-measured in relation to a relatively fast chemical time-for which the $Z$ and $\tilde{T}$ profiles would remain closely associated with a stable manifold around converging streamlines. In this context, the behaviour of the end of the diffusion flame $\{Z \equiv S\} \cap\left\{\tilde{T} \equiv \tilde{T}_{c}\right\}$ is then governed by convection due to the flow as well as possible propagation of the end of the flame along the stoichiometric surface $Z \equiv S$.

The principal surviving influences in the flow-field now lead to the following equations for $Z$ and $\tilde{T}$ :

$$
\begin{aligned}
& \tilde{\sigma}\left(y_{0}-y\right) Z_{y}=Z_{y y} \\
&(\tilde{V}+\tilde{\mu} x+\tilde{\tau} y) \tilde{T}_{x}+\tilde{\sigma}\left(y_{0}-y\right) \tilde{T}_{y}=\tilde{T}_{x x}+\tilde{T}_{y y}+ \\
&+\beta^{4}[Z-S \tilde{T}][1-Z-(1-S) \tilde{T}] \exp \left(\frac{\beta(\tilde{T}-1)}{1+\alpha(\tilde{T}-1)}\right)
\end{aligned}
$$

where $\tilde{\sigma}>0$ is the converging strain-rate in the flow from the normal direction $y, \quad \tilde{V}$ is the propagation speed relative to the gas velocity at $x=y=0, \tilde{\mu} \geq$ 0 is the diverging strain-rate (stretch) in the direction of propagation, and $-\tilde{\tau}$ is the tangential rate of shear in that direction. The stagnation position $y=y_{0}$ is determined by the condition that $Z=S$ at $y=0$.

Without curvature effects or with relatively weak diffusion, any real solution for $Z$ is only stable if $\tilde{\sigma}$ is positive and $0 \leq \tilde{\mu} \leq \tilde{\sigma}$-requiring that there must be no inward convection from within the surface $\{Z \equiv S\}$. All other linear velocity components relate either to changes in the $z$ direction or to convection (including rotation) of the axes. They therefore play no role in this local two-dimensional model. In general, a typical turbulent flow field would tend to generate local values of $\tilde{\sigma}, \tilde{\mu}$ and $\tilde{\tau}$ that should be of a similar order of magnitude at any point and time.

It should be possible to consider higher order velocity components, curvature of the stoichiometric surface and even slow velocity changes, but the dominant influence on the nature of the end of the diffusion flame would normally be contained in these leading order local flow attributes. Significant improvements to the straightforward two-dimensional sub-model ( 8 ) should be based primarily upon an improved understanding of the behaviour of $Z$, and in particular the surface $\{Z \equiv S\}$ in more complicated or curved geometries and flow fields. Some numerical investigations of this type have been carried out, as in reference [28] for example, but a greater theoretical understanding is still being sought. 
This particular representation of the laminar triple-flamelet model arises from the scalings and non-dimensionalisation selected in equations (6) and (7). Any linear rescaling would produce a completely equivalent model. For instance, a rescaling that uses the flow-field to provide a reference velocity (rather than $u_{c}$ ) could be made to replace $\sigma$ by unity and $\beta^{4}$ by a more familiar [5] Damköhler number $D a \propto \sigma^{-1}$. However, the model equations (8) are more suitable for the present discussion because they retain a more direct connection with the basic properties of the flow-field. In this $\tilde{\sigma}, \tilde{\mu}$ and $\tilde{\tau}$ may vary substantially, and the scalings (6) are chosen only in anticipation of something interesting happening at order one values of $\tilde{\sigma}, \tilde{\mu}$ and $\tilde{\tau}$.

The relevant solution for $Z$ in the model (8) can be written in terms of the complementary error function

$$
\begin{gathered}
Z=\frac{1}{2} \operatorname{erfc}\left(\sqrt{\tilde{\sigma} / 2}\left(y_{0}-y\right)\right) \\
\text { giving } \quad \tilde{\chi}(Z, \tilde{\sigma})=Z_{y}{ }^{2}=(\tilde{\sigma} / 2 \pi) \exp \left(-\tilde{\sigma}\left(y-y_{0}\right)^{2}\right)
\end{gathered}
$$

where $\tilde{\chi}=Z_{y}{ }^{2}$ represents a 'scalar dissipation rate' [10] that is directly proportional to the normal strain-rate $\tilde{\sigma}$ at any fixed value of $Z$. The condition $Z\left(y_{0}\right)=S$ now determines $y_{0}(S)$ so that $\chi$ has the separable form $\chi=\tilde{\sigma} h(Z ; S)$. Eliminating $y$ in favour of $Z$ as a new independent variable now yields the single equation

$$
\begin{aligned}
(\tilde{V}+\tilde{\mu} x+\tilde{\tau} y) \tilde{T}_{x} & =\tilde{T}_{x x}+\tilde{\chi} \tilde{T}_{Z Z}+ \\
& +\beta^{4}[Z-S \tilde{T}][1-Z-(1-S) \tilde{T}] \exp \left(\frac{\beta(\tilde{T}-1)}{1+\alpha(\tilde{T}-1)}\right)
\end{aligned}
$$

which needs to be solved subject to the boundary conditions

$$
\begin{gathered}
\tilde{T}(\infty, Z)=\min \{Z / S,(1-Z) /(1-S)\}-O\left(\beta^{-1}\right), \\
\tilde{T}(-\infty, Z)=O\left(\beta^{4} e^{-\beta}\right), \quad \tilde{T}(x, 0)=\tilde{T}(x, 1)=0 \quad \text { and } \quad \tilde{T}(0, S)=\tilde{T}_{c}
\end{gathered}
$$

in which the functional form $\tilde{T}=\tilde{T}(x, Z)$ is assumed. This steady two-dimensional triple-flame problem can now be studied in the useful asymptotic limit $\beta \rightarrow \infty$.

\section{Triple-Flame Behaviour.}

Equation (9) shows that the stratification gradient $Z_{y}$ of the mixture varies in proportion to the square root of $\tilde{\sigma}$ (or $\tilde{\chi}$ ). Thus, since it is primarily $Z_{y}$ that influences the structure and propagation speed of any triple-flame [22], one finds that different regimes of behaviour can be found for different ranges of values of $\tilde{\sigma}>0$. The full range of behaviour of triple flames, arising from the equidiffusive model that has just been presented, is discussed in the following sections.

3.1 Forwards Propagation. For sufficiently small values of $\tilde{\sigma}$ (along with $\tilde{\mu}$ and $\tilde{\tau}$ ), the stratification of the mixture can become so weak that the fuelrich and fuel-lean premixed flames in the triple-flame structure become almost onedimensional. The leading end of the triple-flame then resembles a plane one-dimensional flame-front propagating into a uniform near-stoichiometric mixture. This regime 
arises for values of strain-rate in the range $\tilde{\sigma}=o\left(\beta^{-1}\right) \ll 1$ and has been analysed in terms of weakly-curved flame fronts [21,22].

In fact $\tilde{\sigma}, \tilde{\mu}$ and $\tilde{\tau}$ are small enough in this regime for their role in the equations to be neglected except in so far as they determine the gradient $Z_{y}=\tilde{\chi}^{1 / 2}$ at $y=0$. The equation governing the behaviour of temperature can then be rewritten in the model form

$$
\begin{aligned}
& \qquad \tilde{V}^{2}\left(\tilde{T}_{X}-\tilde{T}_{X X}-\tilde{T}_{Y Y}\right)=\beta^{4}[Z-S \tilde{T}][1-Z-(1-S) \tilde{T}] \exp \left(\frac{\beta(\tilde{T}-1)}{1+\alpha(\tilde{T}-1)}\right) \\
& \text { where } \quad X=\tilde{V} x \quad \text { and } \quad Y=\tilde{V} y \\
& \text { with } \quad Z=S+B Y / \beta+O\left(B^{2} / \beta^{2}\right) \quad: \quad B=\beta \sqrt{\tilde{\chi}_{s}} / \tilde{V}
\end{aligned}
$$

where $\tilde{\chi}_{s}=\tilde{\chi}(S, \tilde{\sigma})$. The problem thus reduces to one already studied in references [21]-[23].

One finds that the propagation speed of the triple-flame is close to the maximum adiabatic laminar flame speed that can be attained by adjusting the value of $Z$ in a uniform mixture. With the scalings defined in equations (6), the propagation speed $\tilde{V}$ is found [22] to be of the order of $\beta^{1 / 2}$ and to vary in the manner

$$
\tilde{V} / \beta^{1 / 2}=V_{0}-V_{1} B+V_{2} B \sqrt{B}+O\left(B^{2}\right)
$$

for $B \ll 1$. In this, $V_{0}, V_{1}$ and $V_{2}$ are order one constants that depend only on chemical parameters (most particularly, on the value of $S$ ).

For increased strain rates in the range $\tilde{\sigma}=O\left(\beta^{-1}\right) \ll 1$, or $B=O(1)$, the premixed flames lose their almost planar character, and one has to take into account an essentially two dimensional nature of the preheating regions of these flames. Nevertheless, the thin chemically active 'reaction zones' of the premixed flames are still found to extend over values of $y$ that are comparable with the thickness of the preheating regions. For the constant density model (7), this type of behaviour has been analysed [23] using an appropriate Green's function to express the problem in the form of an integral equation. Numerical solutions reveal that $\tilde{V}$ remains positive and of the order of $\beta^{1 / 2}$ as $\tilde{\sigma}$ increases, although $\tilde{V} / \beta^{1 / 2}$ decreases monotonically as $B$ (or $\tilde{\sigma}$ ) is increased. For the sample case $S=\frac{1}{2}$, this variation is reproduced in figure 3.

3.2 Positive and Negative Propagation Speed. As $\tilde{\sigma}$ is increased further, the reaction zones associated with the premixed parts of the triple-flame structure move closer and closer to the diffusion flame until, for $\tilde{\sigma}=O(1)$, their extent in $y$ becomes very small compared with the extent of any region of preheating. A brief examination of this regime of behaviour has been carried out [16] and is discussed in some more detail below. In terms of an asymptotic analysis, the solution structure can be divided into several matching parts.

Chemically-Frozen Region: Since the reaction is only really significant where $\tilde{T}=1-O\left(\beta^{-1}\right)$, an outer asymptotic problem as $\beta \rightarrow \infty$ can be identified in terms of the asymptotic form $\tilde{T} \sim T_{0}(x, Z)<1$, the domain of which must exclude the line $Z=S$ for all $x \geq 0$ where a distribution of chemical heat sources is to be found. 


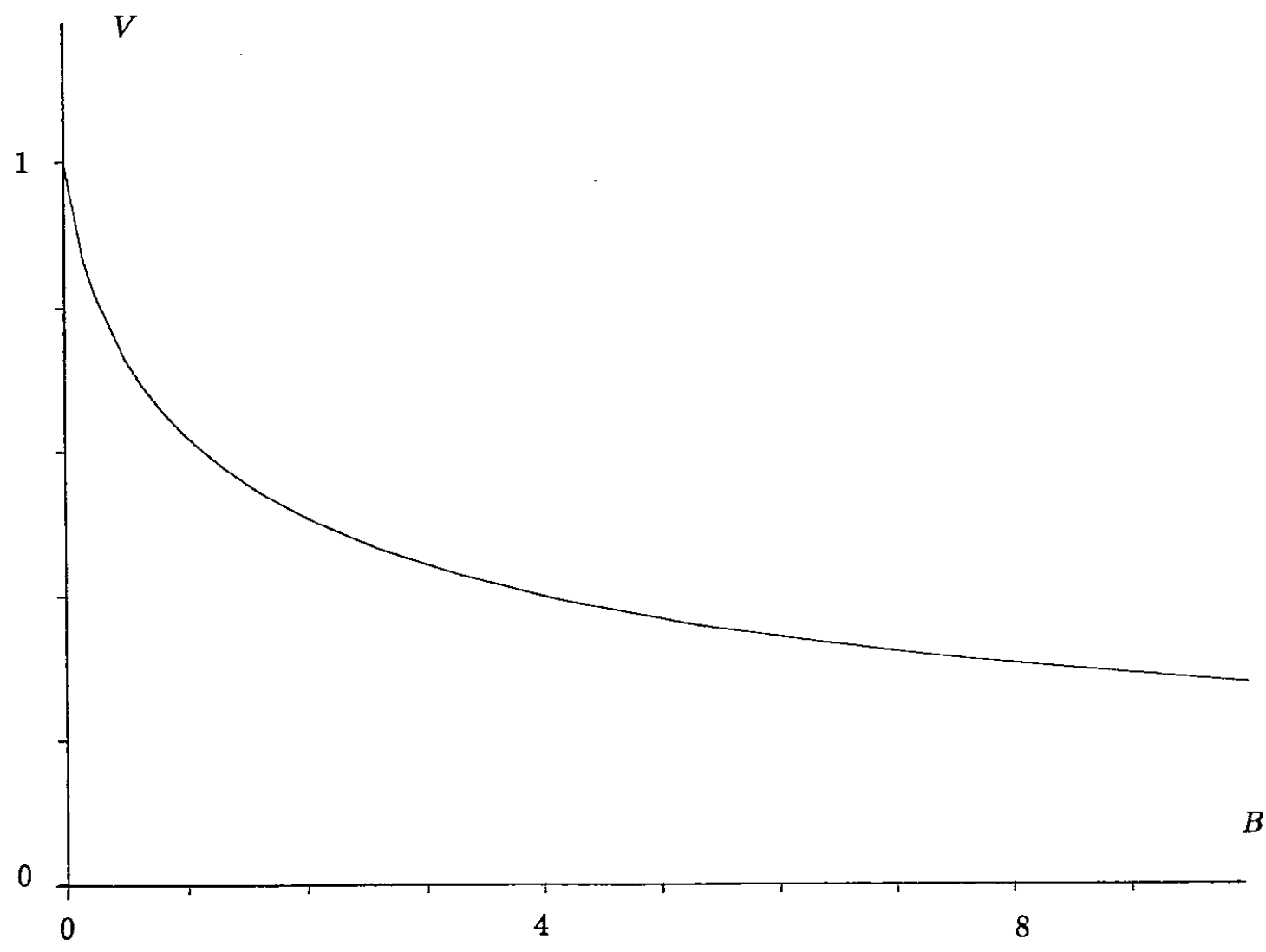

Figure 3. Propagation speed of a symmetric triple-flame (having $S=\frac{1}{2}$ ) when $\tilde{\sigma}=$ $O\left(\beta^{-1}\right)$, reproduced from [23].

This leads to the equation and boundary conditions

$$
(\tilde{V}+\tilde{\mu} x+\tilde{\tau} y) T_{0_{x}}=T_{0 x x}+\tilde{\chi} T_{0 Z Z}
$$

$$
\begin{array}{ll}
\text { with } & T_{0}(\infty, Z)=\min \{Z / S,(1-Z) /(1-S)\}, \quad T_{0}(-\infty, Z)=O \\
& T_{0}(x, 0)=T_{0}(x, 1)=0, \quad \text { and } T_{0}\left(x, S^{ \pm}\right)=1 \text { for } x \geq 0 .
\end{array}
$$

It may be noted that only the asymptotic analysis of this outer region would need to be modified if the small heat release assumption were to be dropped.

Because of the presence of the line source ending at $x=0$ and $Z=S$ or $y=0$, the solution to the problem (14) inevitably involves a square-root singularity at $(x, Z)=(0, S)$ of the form

$$
T_{0} \sim 1-K(r-x)^{1 / 2}=\operatorname{Re}\left\{1+\sqrt{2} i K(x+i y)^{1 / 2}\right\}
$$

$$
\text { with } \quad r=\left(x^{2}+y^{2}\right)^{1 / 2} \sim\left(x^{2}+(Z-S)^{2} / \tilde{\chi}_{s}\right)^{1 / 2} \text {. }
$$

An isometric diagram showing the shape of the solution $T_{0}(x, y)$, including its singular nature near $x=y=0$, is presented in figure 4 . The constant $K$ would form 
part of any solution obtained for $T_{0}$, and would need also to tie in (or match) with a corresponding square-root behaviour found by considering an 'inner region' in which significant chemical activity takes place.

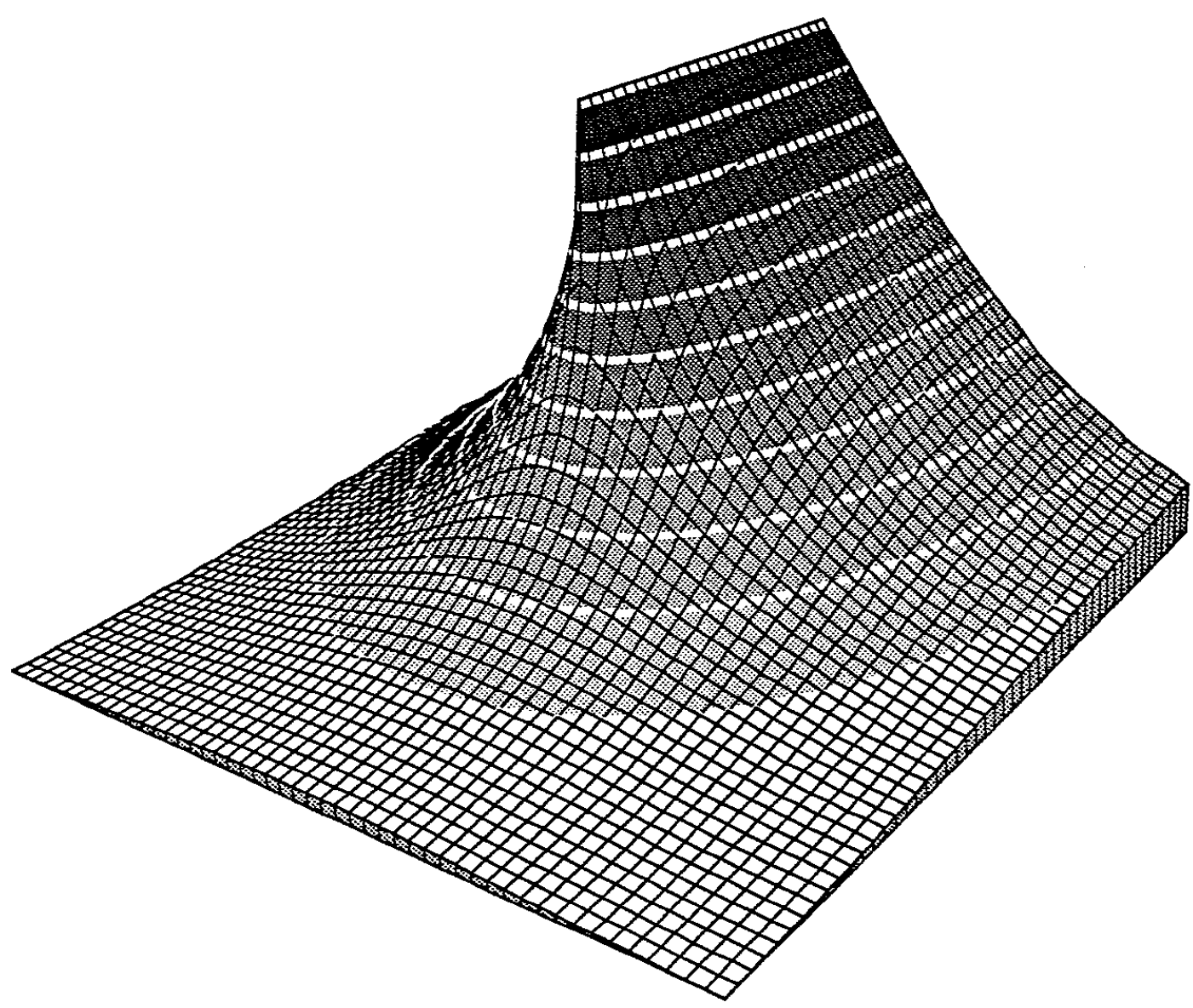

Figure 4. An isometric diagram of the outer temperature solution in the case $\tilde{\sigma}=1$, $\tilde{V}=1$ and $\tilde{\mu}=\tilde{\tau}=0$ over the range $x \in[-1.5,1.5]$ with $y \in[-1.5,1.5]$.

Premixed Flame-Sheet: The chemical activity in the neighbourhood of $Z=S$ is initiated by the premixed flame-sheets of a triple-flame. Defining the rescaled variables $\zeta=(Z-S) \beta$ and $\xi=x \beta \tilde{\chi}_{S}^{1 / 2}$, these can be considered to follow a path, $\xi+i \zeta=W(\ell)$ (for any suitable parametric variable $\ell$ ) at which it is convenient to identify a real-valued local normal coordinate $\eta \in \Re$, defined by $\xi+i \zeta=W+$ $i W_{s} \eta / \beta^{1 / 2}$ where $W_{s}$ is the complex tangential direction $W^{\prime}(\ell) /\left|W^{\prime}(\ell)\right|$. The local asymptotic temperature structure $\tilde{T} \sim 1-\beta^{-1} \phi_{0}(\eta, \zeta)$ is then found to be described 
by

$$
\begin{array}{cc}
\tilde{\chi}_{S} \phi_{0 \eta \eta}= & {\left[S \phi_{0}+\zeta\right]\left[(1-S) \phi_{0}-\zeta\right] \exp \left(-\phi_{0}\right)} \\
\Longrightarrow \quad\left[\tilde{\chi}_{s} \phi_{0 \eta}^{2}\right]_{-\infty}^{\infty}=\Omega^{2}=2 \int_{\Gamma_{0}(\zeta, S)}^{\infty}[S \gamma+\zeta][(1-S) \gamma-\zeta] \exp (-\gamma) d \gamma \\
\text { with } \\
\qquad \Gamma_{0}(\zeta, S)=\max \left\{-\frac{\zeta}{S}, \frac{\zeta}{1-S}\right\}
\end{array}
$$

after using the boundary condition that the reaction-rate term must tend to zero behind the flames. This shows that the function $\Omega(\zeta, S)$ determines a jump condition in the gradient of $\tilde{T}$ across the flame-path, namely $\left[\left|\left(\tilde{T}_{\xi}, \tilde{T}_{\zeta}\right)\right|\right]_{-} \sim\left(\beta^{-1} / \tilde{\chi}_{s}\right)^{1 / 2} \Omega$.

In the 'preheating' region of this flame, it follows that it is appropriate to identify the asymptotic structure $\tilde{T} \sim 1-\left(\beta^{-1} / \tilde{\chi}_{S}\right)^{1 / 2} \Phi(\xi, \zeta)$, which satisfies the equation and matching requirements

$$
\begin{gathered}
\Phi_{\xi \xi}+\Phi_{\zeta \zeta}=0, \\
\text { with } \Phi \equiv 0 \text { and } \Phi_{\xi}-i \Phi_{\zeta}=-i W_{s}^{*} \Omega(\zeta, S) \text { on } \xi+i \zeta=W(\ell) .
\end{gathered}
$$

Because the temperature perturbation $\Phi$ is a harmonic function of $\xi+i \zeta$ it can thus be shown that the flame-path $W(\ell)$ must satisfy the nonlinear principal value integral equation

$$
\Omega(\operatorname{Im}\{W(\ell)\}, S)=\frac{1}{\pi} f \Omega(\operatorname{Im}\{W(\gamma)\}, S) \operatorname{Im}\left\{\frac{W_{s}(\ell)}{W(\gamma)-W(\ell)}\right\}\left|W^{\prime}(\gamma)\right| d \gamma
$$

In this, integration is taken over a clockwise contour consisting of the entire flamepath, for all values of the parameter $\ell$. One flame-path solution of this equation is shown in figure 5 for the symmetric case $S=\frac{1}{2}$.

It is interesting to note that equation (18) has a universal character that depends only on the reaction-zone jump-condition function $\Omega(\zeta, S)$ and the requirement that relatively small overall temperature changes take place along the reaction-sheet. A similar equation may therefore apply, even for systems involving complicated chemical processes, as soon as a suitable function $\Omega$ can be determined. All of the effects of thermal expansion, changes in thermal conductivity etc. are significant only in the outer domain so that a similar analysis of the inner domain may still be found even if these effects were to be included fully in the model.

In general, the flame path $\xi+i \zeta=W(\ell)$ appears to be unique for a given reaction function $\Omega$. At large values of $\xi+i \zeta$ one must also find that

$$
\Phi_{\xi}-i \Phi_{\zeta}=\frac{1}{2 \pi} \oint \Omega(\operatorname{Im}\{W(\gamma)\}, S) \frac{\left|W^{\prime}(\gamma)\right| d \gamma}{\xi+i \zeta-W(\gamma)} \sim-L \frac{i}{\sqrt{2}} /(\xi+i \zeta)^{1 / 2}
$$

which mimics the square-root singular behaviour (15) of the outer problem. In the symmetric case, $S=\frac{1}{2}$, the value of the constant $L(\Omega)$ is found to be $L \approx 0.7945$.

Matching and Propagation Speed: The constant $L$ thus depends only on the structure of the reaction function $\Omega(\zeta, S)$. Matching of the result (19) with the singular part (15) of the solution of the outer problem requires that

$$
K=L / \tilde{\chi}_{s}^{1 / 4}
$$




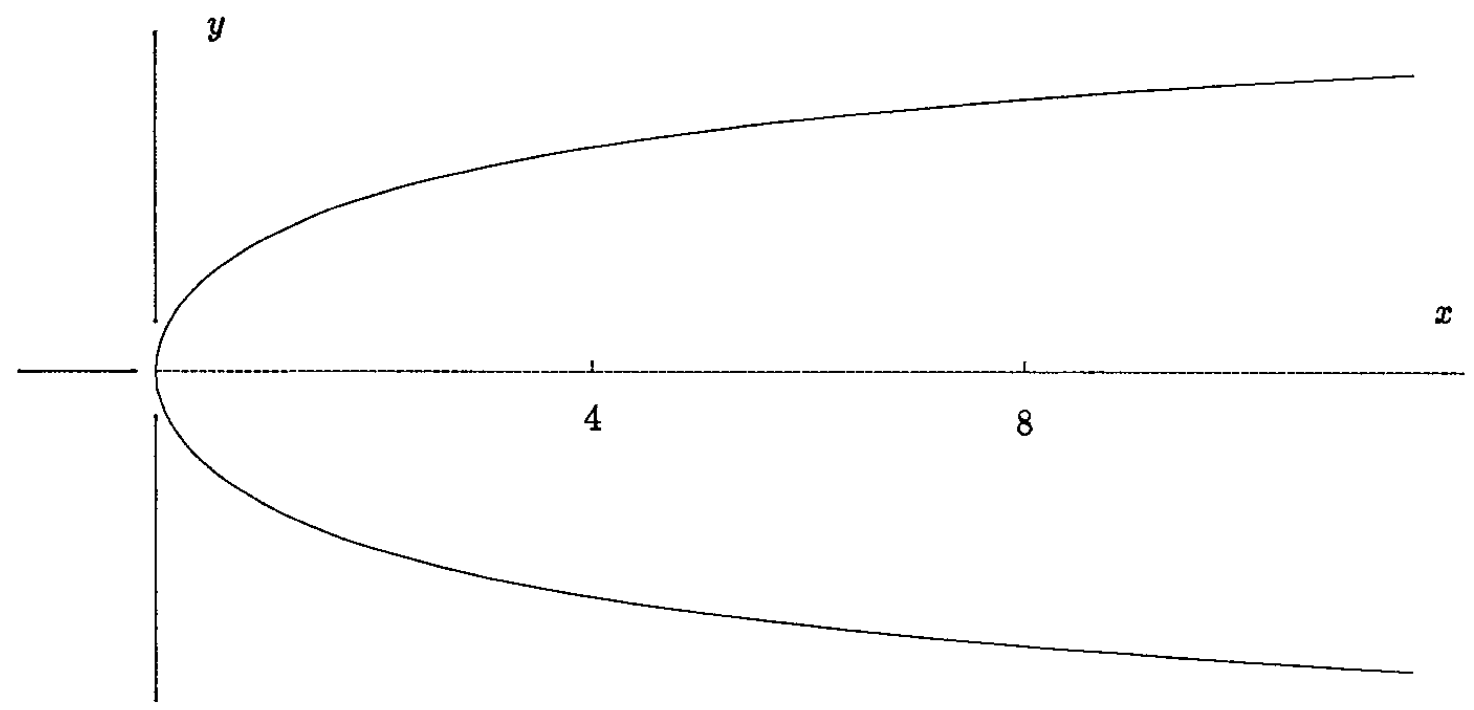

Figure 5. Premixed flame paths (shown as a solid curve) and the diffusion flame path (shown dashed) in the symmetric case $S=\frac{1}{2}$. The $x$ and $y$ axes are scaled equally to reveal the actual physical shape of the triple-flame structure.

which thus fixes the allowed value of $K(\tilde{\sigma}, \tilde{\tau}, \tilde{\mu}, \tilde{V}, S)$ for a given set of the flow attributes $\tilde{\sigma}, \tilde{\mu}$ and $\tilde{\tau}$ and the stoichiometric constant $S$. In general, the outer problem only yields a suitable value of $K$ for 'eigenvalue' combinations of the arguments of $K$ so that, in particular, the propagation speed $\tilde{V}$ becomes a function of the form $\tilde{V}=\tilde{V}(\tilde{\sigma}, \tilde{\tau}, \tilde{\mu}, S)$.

Considering (say) the case with $S=\frac{1}{2}$ and $\tilde{\mu}=\tilde{\tau}=0$, the speed of propagation can thus be thought of as being determined by fixed contour values of $L=K \tilde{\chi}_{S}^{1 / 4}$ in the domain of $(\tilde{V}, \tilde{\sigma})$. For illustration, some numerically calculated contours of this type are presented in figure 6 in which the dotted curve represents the contour of $L \approx 0.7945$. As a result, this reveals the way in which the propagation speed $\tilde{V}$ varies with $\tilde{\sigma}$ for $S=\frac{1}{2}$. Qualitatively similar results are found for different chemical parameters.

More generally, the propagation speed $\tilde{V}$ is found to be positive for all small enough values of $\tilde{\sigma}, \tilde{\tau}$ and $\tilde{\mu}$ (as described previously), but it also becomes zero for a particular combination $\tilde{\sigma}=\tilde{\sigma}_{0}(\tilde{\tau}, \tilde{\mu}, S)$. Larger values of $\tilde{\sigma}$ result in negative 


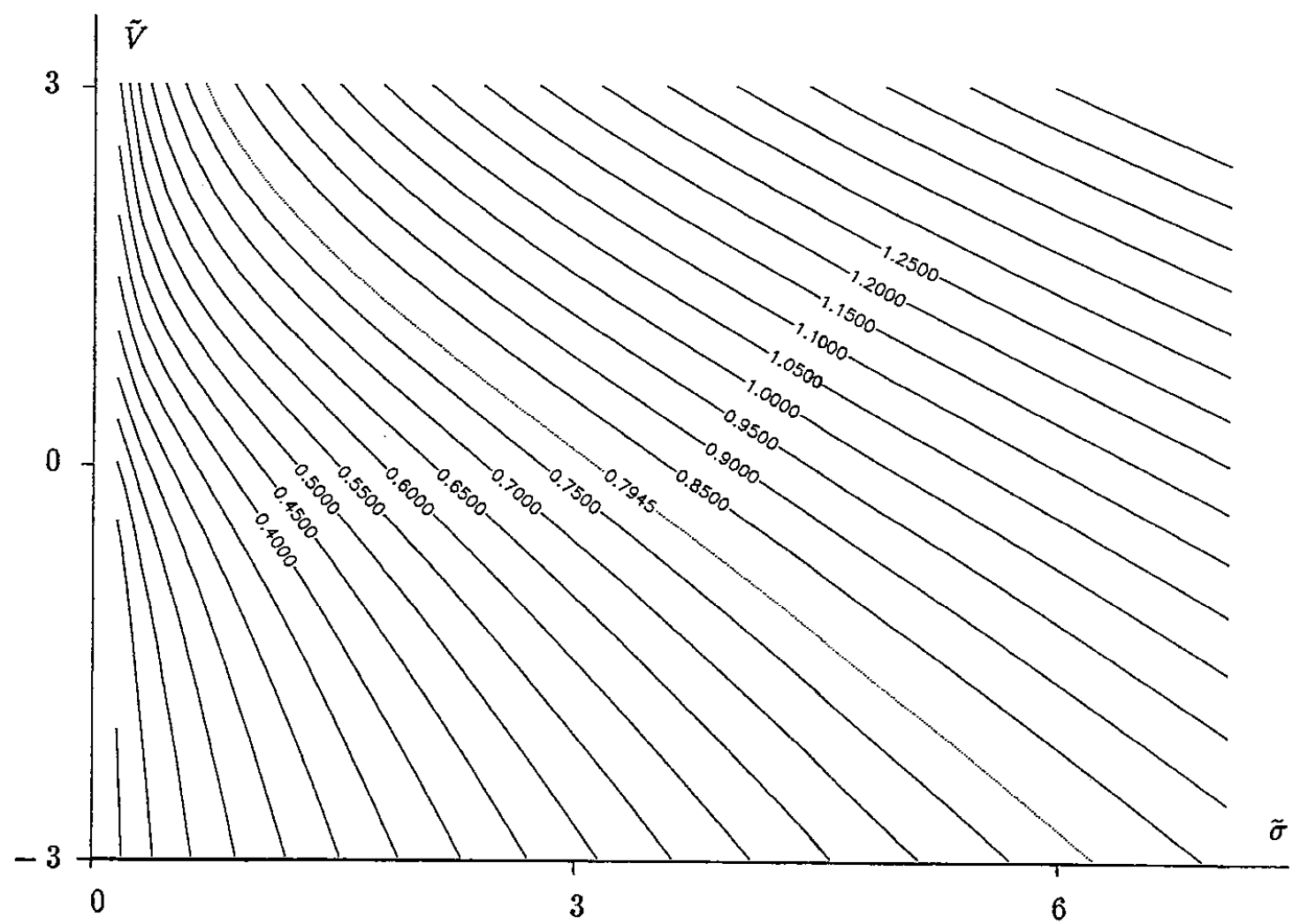

Figure 6. Contours of fixed values of $L=K \chi_{s}^{1 / 4}$ over a part of the domain of $(\tilde{V}, \tilde{\sigma})$ in the case having $\tilde{\mu}=\tilde{\tau}=0$ and $S=\frac{1}{2}$.

propagation-speeds-that is, the end of the diffusion-flame recedes rather than advances and a front of extinction propagates into the diffusion flame-sheet. In order to understand the reason for this, it is necessary only to recognise that points near an end of a diffusion flame (or any hot surface in a cooler atmosphere) are typically subjected to much greater heat losses than points in any interior section. Unless chemical and thermodiffusive effects are able to compensate for these increased losses, an unsteady cooling (and hence extinction) of the chemical activity at the end point would ensue. The end of the diffusion flame is thus moved further into the flame and can continue to move as a wave of chemical extinction.

3.3 Fast Propagation of an Extinction Front. For strain rates that are increased still further, a point is eventually reached at which no steady uniform diffusion flame can be sustained [5]. Chemical and thermodiffusive effects become unable to overcome heat losses at any position in a diffusion flame, not only near an end point, so that the entire flame extinguishes. In terms of the flow attributes $g$, this happens when $g$ enters the extinction domain $\mathcal{D} \backslash \mathcal{D}_{B}$ by crossing the boundary $\partial \mathcal{D}_{B}$.

Since a steady uniform diffusion flame is in effect a function of $y$ only, the only 
component of $g$ that plays a significant role in determining the extinction boundary $\partial \mathcal{D}_{B}$ is the converging strain-rate $\tilde{\sigma}$. In terms of the dimensionless equidiffusive model equation (10), a uniform diffusion flame extinguishes at the relatively large critical strain-rate $\tilde{\sigma}=\tilde{\sigma}_{c}(\beta, S)=O(\beta)$, as demonstrated below. For $\tilde{\sigma} \geq \tilde{\sigma}_{c}$, no two-dimensional triple-flame solution can exist that satisfies the boundary conditions (11) primarily because the assumption of a steady uniform diffusion flame behind the triple flame (represented by the condition at $x=+\infty$ ) cannot be sustained.

As a result, triple flame solutions for $\tilde{T}$ and $\tilde{V}$ do not exist for $\tilde{\sigma} \geq \tilde{\sigma}_{c}$. For values of the strain rate in the range $1 \ll \tilde{\sigma}<\tilde{\sigma}_{c}=O(\beta)$, with $\tilde{\mu}$ and $\tilde{\tau}$ assuming similar orders of magnitude, the equidiffusive model leads to a large negative speed of propagation. In particular, as $\tilde{\sigma}$ increases towards $\tilde{\sigma}_{c}$, the propagation speed $\tilde{V}$ approaches a finite negative limit. For lower values of $\tilde{\sigma}$ in the range $\tilde{\sigma}=O\left(\tilde{\sigma}_{c}\right)$, one finds that $\tilde{V}=-O\left(\beta^{3 / 2}\right)$. Since this velocity is much greater than the typical range of values hypothesised for $\tilde{\mu}$ and $\tilde{\tau}$, one still finds that the behaviour of the thermal and chemical field is only significantly influenced by the single component $\tilde{\sigma}$ of the vector of flow attributes $\boldsymbol{g}$.

In order to investigate these assertions, one needs to examine the structure of the end of a diffusion flamelet for strain and shear rates $\tilde{\sigma}, \tilde{\mu}$ and $\tilde{\tau}$ of the same order of magnitude as $\beta \gg 1$. This can be done by defining

$$
D=\beta / \tilde{\chi}_{S} \propto \tilde{\sigma}_{c} / \tilde{\sigma}, \quad A=\beta^{3} /\left(\tilde{V}^{2} D\right) \text { and } \quad x=-\lambda \tilde{V} D / \beta^{3}
$$

along with the asymptotic form $\tilde{T} \sim 1-\beta^{-1} \theta(\lambda, \zeta)$. The equation satisfied by the temperature perturbation $\theta$ is then

$$
\theta_{\lambda}+A \theta_{\lambda \lambda}+\theta_{\zeta \zeta}=D[S \theta+\zeta][(1-S) \theta-\zeta] \exp (-\theta)
$$

Interestingly, $-\lambda$ adopts the role of a time coordinate in this equation in the limit as $A$ tends to zero or $\tilde{V}^{2}$ tends to infinity. Physically, even though the downstream heat loss term $A \theta_{\lambda \lambda}$ is crucial in bringing about any extinction of the chemical reaction, its value becomes small when $-\tilde{V}$ is large. The situation then represents the gradual cooling of gases as they are rapidly convected past a region of chemical extinction.

Appropriate boundary conditions for this problem are most usefully posed in terms of a function $\tilde{\theta}(\zeta ; D)$ that is defined to satisfy the equation and boundary condition

$$
\begin{aligned}
\tilde{\theta}_{\zeta \zeta} & =D(S \tilde{\theta}+\zeta)((1-S) \tilde{\theta}-\zeta) \exp (-\tilde{\theta}) \\
\text { with } \quad \tilde{\theta} & =\Gamma_{0}(\zeta, S)+O(1) \text { as } \zeta \rightarrow \pm \infty .
\end{aligned}
$$

Solutions of this problem are only found to exist for $D \geq D_{c}(S)=O(1)$, or equivalently $\tilde{\sigma} \leq \tilde{\sigma}_{c}=O(\beta)$ from the definition (21) of $D$. Moreover, for $D>D_{c}$, two solutions are found representing either a hot diffusion flame or a cooler 'partially premixed' flame [5]. Of these, we select the stable hotter solution, corresponding to lower values of $\tilde{\theta}$. Once such a solution has been found, the boundary conditions for $\theta$ can be stated in the form

$$
\begin{gathered}
\theta(\lambda, \zeta) \sim \tilde{\theta}(\zeta ; D) \text { as } \lambda \rightarrow \infty \text { and/or } \zeta \rightarrow \pm \infty \\
\theta(-\infty, \zeta)=\infty \text { and } \theta(0,0)=\theta_{c} .
\end{gathered}
$$


where $\theta_{c} \geq \tilde{\theta}\left(0 ; D_{c}\right)$ representing the temperature at the stoichiometric surface in a uniform diffusion flame at the critical strain-rate for extinction, or any convenient larger value corresponding to $T_{c}$. The last of these conditions mainly serves to eliminate any translational invariance in the problem.

Clearly, appropriate solutions for $\theta(\lambda, \zeta)$ are only possible in the range $D>D_{c}$, and then only for particular values of the parameter $A=A(D)$. These order one values of $A$ reveal that the 'propagation' speed $\tilde{V}$ would now be negative and of the order of magnitude $\tilde{V}=-O\left(\beta^{3 / 2}\right)$ as indicated earlier. Moreover, as $D$ decreases towards $D_{c}$, the steady diffusion flame solution (represented by $\tilde{\theta}$ at $\lambda=\infty$ ) is more easily extinguished by smaller heat losses $A \theta_{\lambda \lambda}$. In fact, one finds that $A$ decreases to a finite critical value $A_{c}(S)$, as $D \rightarrow D_{c}$, at which a turning point bifurcation arises to an unphysical branch of solutions that have corresponding unstable diffusion-flame solutions of (23) at their burnt boundaries. This shows that $\tilde{V} \rightarrow \tilde{V}_{c}=-O(\beta 3 \hat{/ 2})$ as $\tilde{\sigma} \rightarrow \tilde{\sigma}_{c}$.

Since the scalings (21) ensure that $\theta_{\lambda \lambda}$ does not vanish in this limit, this makes it clear that one should expect $A$ to decrease towards zero, showing that $\tilde{V} \rightarrow-\infty$ as $\tilde{\sigma} \rightarrow \tilde{\sigma}_{c}$.

3.4 Range of Propagation Speeds. Writing $V_{L}$ and $\sigma(\boldsymbol{r}, t)$ for the dimensional values of the maximum laminar flame speed (obtainable by varying $Z$ ) and of the converging strain-rate, respectively, the general behaviour of the dimensional triple-flame propagation speed $V$ can be summarised as follows. With $\sigma_{c}$ denoting the critical strain-rate for extinction of a uniform diffusion flame (corresponding to $\tilde{\sigma}_{c}$ ), the propagation speed $V$ depends almost exclusively on $\sigma$ in the ranges $\sigma \gg \sigma_{c} / \beta$ and $\sigma \ll \sigma_{c} / \beta$.

In both of these ranges, this finding depends on assuming that other velocity-field components, such as the (dimensional) shear-rate $\tau$ and the diverging strain-rate $\mu$ in the direction and plane of triple-flame propagation, are similar in magnitude to the normal strain-rate $\sigma$. Although the magnitudes of these components are likely to be strongly correlated, this need not exclusively be the case in a turbulent flow. For relatively strong rates of shear and vorticity that produce flow velocity components as large as $V$ in the domain of a triple-flame these effects could not be ignored. However, the propagation speed $V$ would still be considerably more sensitive to changes in $\sigma$ than to changes in these other quantities. In the range, $\sigma=O\left(\sigma_{c} / \beta\right)$, flow velocities caused by $\sigma, \mu$ and $\tau$ are all similar in magnitude to typical values of the propagation speed $V$ that might be encountered. Thus $V$ is likely to be comparably sensitive to all of these flow properties.

In the smaller range, particularly when $\sigma=O\left(\sigma_{c} / \beta^{2}\right), V$ is found to be positive and of the order of $V_{L}$, approaching $V_{L}$ as $\beta^{2} \sigma / \sigma_{c}$ decreases towards zero. If the model were to be extended to include thermal expansion, then $V$ would approach a larger value than $V_{L}$ in this limit [17] because of the Landau effect [29, p. 487]. In the larger range, $\sigma_{c} / \beta \ll \sigma<\sigma_{c}$, it is found that $V$ becomes relatively large and negative (corresponding to a receding flame), being of the order of magnitude $V=-O\left(\beta V_{L}\right)$. Moreover, as $\sigma$ approaches the critical value $\sigma_{c}$, the speed $V$ decreases to a negative lower limit $V_{c}=-O\left(\beta V_{L}\right)<0$. 
In the intermediate range, $\sigma=O\left(\sigma_{c} / \beta\right)$, the propagation speed becomes relatively small in magnitude, now falling into the range $V=O\left(V_{L} / \beta^{1 / 2}\right)$. As described above, it is now also found to be affected significantly by other flow attributes such as $\mu$ and $\tau$. Most significantly, it is in this range that $V$ changes sign at some value of $\sigma=\sigma_{0}=O\left(\sigma_{c} / \beta\right)$ that depends on the other flow attributes as well, of course, as on the properties of the chemical reaction, $\sigma_{0}=\sigma_{0}(\mu, \tau ; S)$. A qualitative sketch of the variation of $V$ with $\sigma$ is presented in figure 7 .

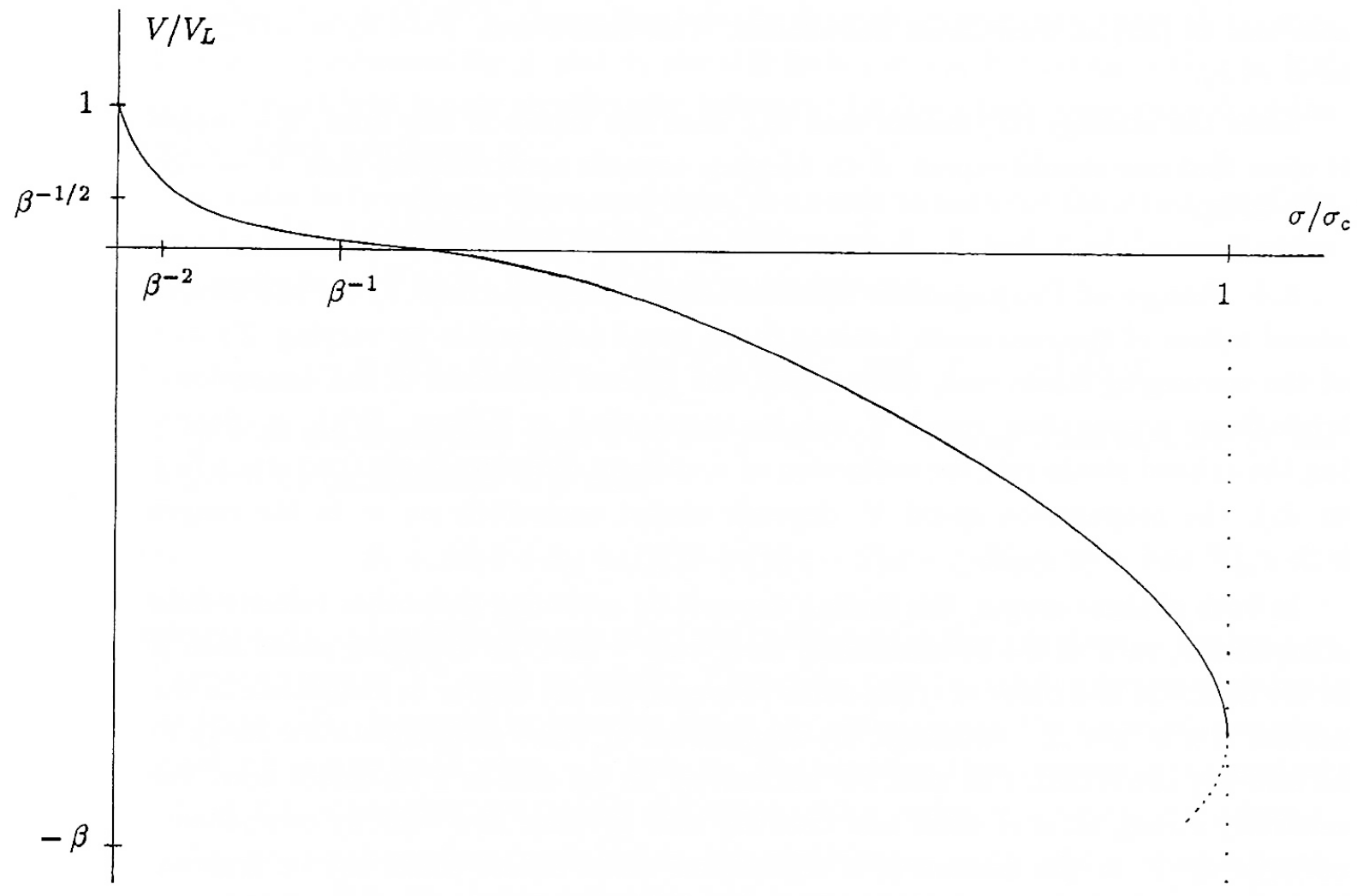

Figure 7. Qualitative sketch of the overall variation of the propagation speed of an equidiffusive triple-flame with changes in flow strain-rate at constant density.

\section{4. 'Holes' in Turbulent Diffusion Flames.}

The inclusion of findings such as these in a description of turbulent diffusion flames is not a straightforward task and the following discussion only provides a number of preliminary ideas. The main intention is to reveal the essential mechanisms that might cause part of a mixing surface to be burning and part to be extinguished in a turbulent diffusion flame.

Modelling such a process must include several significant components that may 
be discussed in terms of a particular realisation. For this purpose one may consider the stoichiometric surface $\mathcal{S}=\{Z \equiv S\}$ to be a known function of time, and the vector of flow attributes $g$ to be a corresponding known continuous function of space and time. Even though a triple-flame may not actually exist at the point $r$ at a time $t$, the propagation speed that such a flame would have at that point $V$ is then also a known function of space and time provided $g \in \mathcal{D}_{B}$. Outside this domain a diffusion flame cannot exist and $V$ is undefined. Within the surface $\mathcal{S}$ one can therefore envisage contours of constant $V$ that change with time, as does the surface itself. For the purpose of discussion it is convenient also to identify the domain of flow attributes $g \in \mathcal{D}^{+} \subset \mathcal{D}_{B}$ in which propagation speeds would be positive $V>0$ at least in the equidiffusive case.

Let us now suppose that a diffusion flame exists throughout some part of $\mathcal{S}$ in which a local minimum value of $V$ decreases towards $V_{c}$ until, at some moment, a contour of $V=V_{c}<0$ appears. 'Inside' this contour $V$ becomes undefined as values of $g$ cross the extinction boundary $\partial \mathcal{D}_{B}$. A brief extinction transient must then ensue, causing a hole (represented by $\mathcal{H} \subset \mathcal{S}$ ) to appear in the diffusion flame. Once this happens, and a triple-flamelet forms around the edges of the hole $\partial \mathcal{H}$, it is straightforward to show that the area $H$ of the hole within the stoichiometric surface grows for a time according to the formula

$$
\frac{d H}{d t}=\iint_{\mathcal{H}} \sigma|d \boldsymbol{A}|-\oint_{\partial \mathcal{H}} V d s .
$$

The area integral in this expression arises from the stretching of the stoichiometric surface as a result of the converging normal flow strain-rate while the line integral around the entire boundary of the hole arises from the propagation of the edge of the hole in the form of a triple-flame.

Recalling that $V$ is initially very large and negative (in comparison to the magnitude of local convective effects) this shows that the diffusion flame pops like a balloon as soon as local flow attributes become intense enough to cause an extinction at some point. The hole $\mathcal{H}$ grows rapidly in size (relative to the underlying surface $S$ ) until its edges approach a countour at which $V=0$ (assuming that such a contour exists). Subsequent changes would tend to involve some dynamical variations in the position of the edge of the hole $\partial \mathcal{H}$ as it is convected within the stoichiometric surface, approximately following the movement of the contour $V=0$. The hole will not be able to close up again until the contour of $V=0$ collapses and disappears.

There is, consequently, some hysteresis in the behaviour of holes in diffusion flames. To come into existence, the flow attributes $g$ must cross the extinction boundary $\partial \mathcal{D}_{B}$, and leave the domain $\mathcal{D}_{B}$ at least briefly in some small part of the stoichiometric surface $\mathcal{S}$. However, once a hole has formed, it can only close up again if values of $g$ decrease so as to produce positive propagation speeds (by entering the smaller domain of values $g \in \mathcal{D}^{+} \subset \mathcal{D}_{B}$ ) uniformly throughout the domain of the hole $\mathcal{H}$. To clarify the dynamical aspects of this hysteresis it now becomes useful to identify three distinct (moving) parts of the surface $\mathcal{S}$. These can be denoted by $\mathcal{S}^{+}$ where $V \geq 0$, by $\mathcal{S}^{-}$where where $V_{c} \leq V \leq 0$ and by $\mathcal{S}_{E}$ where $V$ is undefined and no diffusion flame can persist. Boundaries between these regions are $\mathcal{S}_{0}=\mathcal{S}^{+} \cap \mathcal{S}^{-}$ and $\mathcal{S}_{c}=\mathcal{S}^{-} \cap \overline{\mathcal{S}_{E}}$, there being no boundary between $\mathcal{S}^{+}$and $\mathcal{S}_{E}$. 
A diffusion flame can then exist only throughout the domain $\mathcal{S}_{F}=\mathcal{S}^{+} \cup \mathcal{S}^{-}$and is therefore punctured wherever and whenever the domain $\mathcal{S}_{E}$ appears, rapidly forming a hole $\mathcal{H}$. However, this hole can only close up again to re-establish the diffusion flame if $\mathcal{H} \cap \mathcal{S}^{-}$becomes empty. Generalising $\mathcal{H}$ to represent the set of all non-burning parts of the surface $\mathcal{S}$, this would tend to suggest that a significant correlation may exist between the domain of holes $\mathcal{H}$ and all isolated parts of $\mathcal{S}^{-}$within which either a region of extinction $\mathcal{S}_{E}$ has previously appeared at some time during its existence or within which no flame existed when the isolated region was first formed. The union of all such regions may be denoted by $\mathcal{S}_{H}^{-} \subseteq \mathcal{S}^{-}$.

Ignoring all rapid transients, one should then find that the non-burning part of the surface would at least be given by

$$
\mathcal{H} \supseteq \mathcal{S}_{H}^{-}
$$

to a reasonable degree of approximation. As soon as any region of $\mathcal{S}_{E}$ appears in the surface $\mathcal{S}$, the entire isolated part of $\mathcal{S}^{-}$that surrounds $\mathcal{S}_{c}$ must be added to $\mathcal{S}_{H}^{-}$. Also, if a part of $\mathcal{S}^{-}$appears (through changes in $\boldsymbol{g}$ ) either within or in contact with the non-burning domain $\mathcal{H}$, then this must be added to $\mathcal{S}_{H}^{-}$, emphasising the presence of a hysteresis by the fact that parts of $\mathcal{S}^{-}$may be burning or non-burning depending on their origins and history. In an evolving system, these conditions may be considered to provide an adequate definition of $\mathcal{S}_{H}^{-}$. It follows that the nonburning domain $\mathcal{H}$ depends not only on the negative propagation domain $\mathcal{S}^{-}$but on the topology of $\mathcal{S}^{-}$and on the history of appearances both of the extinction domain $\mathcal{S}_{E}$ and of the domain $\mathcal{S}^{-}$itself within the stoichiometric surface.

Although $V \geq 0$ in the domain $\mathcal{S}^{+}$, it is only possible to say that a flame will tend to propagate and fill the domain $\mathcal{S}^{+}$where such a flame already exists either within or on the boundaries of isolated parts of $\mathcal{S}^{+}$. At one extreme, there may be no burning at all so that $\mathcal{H} \equiv \mathcal{S}$. More generally a contour of $V=0$, surrounding a part of $\mathcal{S}^{+}$, may appear entirely within an extinguished or non-burning part of $\mathcal{S}$. Also, the evolving surface $\mathcal{S}$ need not conserve its connectedness and a non-burning region may become isolated from the rest of $\mathcal{S}$ and subsequently enter the domain $\mathcal{S}^{+}$ as values of $\boldsymbol{g}$ change. In such cases, isolated parts of $\mathcal{H} \cap \mathcal{S}^{+}$, can only be re-ignited by making contact with the burning part of the stoichiometric surface $\mathcal{B}=\mathcal{S} \backslash \mathcal{H}$ ignoring any relatively slow self-ignition transient as $\boldsymbol{g}$ crosses the ignition boundary $\partial \mathcal{D}_{E}$. In an evolving system, a non-burning part of $\mathcal{S}^{+}$may be correlated with a set $\mathcal{S}_{H}^{+}$to which all isolated regions of $\mathcal{S}^{+}$that form entirely within $\mathcal{H}$ are added, and from which any isolated region is removed as soon as any part of its boundary meets a burning diffusion flame, that is $\overline{\mathcal{S}_{H}^{+}} \cap \overline{\mathcal{B}}$ becomes non-empty.

Again ignoring transients, one would find

$$
\mathcal{H} \approx \mathcal{S}_{H}^{+} \cup \mathcal{S}_{H}^{-}
$$

to a moderate degree of approximation. Since the contrast between the magnitude of $V$ and the appropriate convective effects arising from $g$ is not as marked in $\mathcal{D}^{+}$ as it is in the rest of the domain $\mathcal{D}_{B}$, this result is not as good an approximation 
as the inequality (26). Slower positive than negative propagation in a comparatively smaller domain of values of $g$ ensures that transient effects are more significant in causing deviations from equation (27) in the domain $\mathcal{S}^{+}$. Moreover, some positive propagation is required for a triple-flamelet to keep pace with the stretching of the surface $\mathcal{S}$ by the normal strain-rate $\sigma$ indicated in equation (25). Nevertheless, equation (27) should provide a moderately good overall approximation, especially if changes in $\boldsymbol{g}$ happen fairly slowly, although it is likely to underestimate the nonburning surface $\mathcal{H}$.

Determining statistical estimates for $\mathcal{S}_{H}^{+}$and $\mathcal{S}_{H}^{-}$is a complicated task requiring specification of more sophisticated probabilities than appear in equation (1). This is particularly true in relating regions of propagation properties with the history of such regions. Recalling that $\mathcal{S}$ is generally a surface of changing topology, connections between burning and non-burning parts of $\mathcal{S}^{+}$can be established as the surface contorts and different parts of it reconnect. This adds an important 'eddy diffusivity' element to the turbulent flame behaviour that can enhance an overall propagation between mostly burning and mostly unburning parts of the surface. It has been postulated that percolation theory could play a role in determining the overall effectiveness of such connections in maintaining combustion [15]. Ways of improving the ideas introduced here and incorporating them into reasonable statistical models are currently under investigation.

\section{Conclusions.}

The behaviour of a triple-flame, connecting a burning diffusion flame with a region of non-burning, is found to vary considerably as a result of imposed velocity or other conditions. At least for the equidiffusive model studied here the triple flame can have positive and negative flame speeds. Close to conditions which would cause an infinite diffusion flame to extinguish uniformly the triple-flame (and so the end of a diffusion flame) can recede very rapidly.

The detailed study of the behaviour of such flames is relatively new and can involve an intricate asymptotic structure. There is still a great deal to be done in examining problems with more complex chemical schemes as well as different diffusivities and significant chemical and thermal expansion. The outline given here introduces some of these more general aspects but concentrates on the simplest equidiffusive case, illustrating some of the main principles involved.

In a variable flow-field the propagation properties of the triple-flame, as well as extinction conditions for an established diffusion flame, introduce a complex dynamical problem that governs the relationship between burning and non-burning regions in the flow. A hysteresis between conditions for extinction and conditions for reestablishment of a flame demand statistical examinations that must include a description of the history of an evolution as well as reconnections in a topologically changing surface at which burning can take place.

Thus, in modelling non-premixed turbulent combustion, this article illustrates the richness and complexity one can find both in the description of the ends of any diffusion flames and in the dynamical behaviour they introduce to the combustion. 
Using more complex triple-flame models is likely to lead to yet further intricacies in analysing dynamical triple-flame structures.

\section{Acknowledgments.}

The preparation of this article benefitted greatly from discussions with J.F. Clarke and A.A. Liñán whose insights contributed to many of its findings. The ideas it contains were also helped by discussions with J.D. Buckmaster during a visit made possible by the U.S. Air Force Office of Scientific Research. The financial and computational support of the British Science and Engineering Research Council is gratefully acknowledged as is the hospitality of the Institute for Mathematics and its Applications in Minneapolis.

\section{REFERENCES}

[1] J.W. Dold, L.J. Hartley and D. Green, Laminar Triple-flamelet Structures in Nonpremixed Turbulent Combustion, submitted (1990).

[2] S.P. Burke and T.E.W. Schumann, Diffusion Flames, 1st Symp. (Int.) on Combustion, The Combustion Institute, 1928, pp. 2-11.

[3] F.A. Williams, 'Combustion Theory,' 2nd ed., Benjamin/Cummings, California, 1985.

[4]. J.F. Clarke, The Diffusion Flame as a Singular Perturbation Problem, J. Eng. Math. 5 (1971) 179-185.

[5] A.A. LIÑÁN, The asymptotic structure of counterflow diffusion flames for large activation energies, Acta Astronautica 1 (1974) 1007-1039.

[6] B. Rogg, F. Behrendt and J. WARNATZ, Turbulent non-premixed combustion in partially premixed diffusion flamelets with detailed chemistry, 21st Symp. (Int.) on Combustion, The Combustion Institute, 1986, pp. 1533-1541.

[7] M.D. Smooke, A.A. Turnbull, R.E. Mitchell and D.E. Keyes, Solution of TwoDimensional Axisymmetric Laminar Diffusion Flames by Adaptive Boundary Value Methods, in 'Mathematical Modelling of Combustion and Related Topics,' C-M. Brauner and C. SchmidtLaine (Eds.), Martinus Nijhoff, Dordrecht, 1988, pp. 261-300.

[8] N. Peters, Laminar Flamelet Concepts in Turbulent Combustion, 21st Symp. (Int.) on Combustion, The Combustion Institute, 1986, pp. 1231-1250.

[9] R.W. BILGer, Turbulent Diffusion Flames, Ann. Rev. Fluid Mech. 21 (1989) 101-135.

[10] N. Peters, W. Hocks and G. Mohiuddin, Turbulent mean reaction rates in the limit of large activation energies, J. Fluid Mech. 110 (1981) 411-432.

[11] P.C. FIFE, 'Dynamics of Internal Layers and Diffusive interfaces,' SIAM, Philadelphia, 1988.

[12] J.W. DOLD and J.F. Clarke, Ignition and Criticality of a Finite Quantity of Gas Released in the Atmosphere, 20th Symp. (Int.) on Combustion, The Combustion Institute, 1984.

[13] J.W. DoLd, Analysis of Thermal Runaway in the Ignition Process, SIAM Jnl. Appl. Math. 49 (1989) 459-480.

[14] A.A. LIÑ̃́N and A. CREspo, Asymptotic analysis of unsteady diffusion flames for large activation energies, Comb. Sci. Tech. 14 (1976) 95-117.

[15] N. Peters, Local Quenching due to Flame Stretch and Non-Premixed Turbulent Combustion, Comb. Sci. Tech. 30 (1983) 1-17.

[16] J.W. Dold, Flame Propagation in a Mixing Layer, 3rd Int. Seminar on Flame Structure, Kazakhstan, 1989, to appear (Nauka Publishers, Novosibirsk). 
[17] H. Phillips, Flame in a Buoyant Methane Layer, 10th Symp. (Int.) on Combustion, The Combustion Institute, 1965, pp. 1277-1283.

[18] Ö. SAVAŞ and S.R. Gollahaldi, Stability of Lifted Laminar Round Gas-Jet Flame, J. Fluid Mech. 165, (1986) 297-318.

[19] Chiun-Hsun Chen and James S. T'Ien, Diffusion Flame Stabilisation at the Leading Edge of a Fuel Plate, Comb. Sci. Tech. 50, (1986) 283-306.

[20] Y. OHKI and S. Tsuge, in 'Dynamics of Reactive Systems, Part I: Flames and Configurations,' J.R. Bowen, J.C. Leyer and R.I. Soloukhin, Prog. Astronautics and Aeronautics 105 (1986), 233-245.

[21] J.W. Dold, Flame Propagation in a Non-Uniform Mixture: The Structure of Anchored TripleFlames, in 'Dynamics of Reactive Systems Part 1: Flames,' A.L. Kuhl, J.R. Bowen, J.C. Leyer and A. Borisov (Eds.), Prog. Astronautics and Aeronautics 113 (1988), 240-248.

[22] J.W. DoLd, Flame Propagation in a Non-Uniform Mirture: Analysis of a Slowly-Varying Triple-Flame, Combustion and Flame 76 (1989) 71-88.

[23] L.J. HARTLeY and J.W. Dold, Flame Propagation in a Non-Uniform Mixture: Analysis of a Propagating Triple-Flame, Comb. Sci. Tech., to appear.

[24] L.J. Hartley, J.W. Dold, A.A. Liñ́n and J.D. BucKmaster, Flame Propagation in a Non-Uniform Mixture: Positive and Negative Triple-Flame Propagation in a Strained Mixing Layer, to be submitted.

[25] A.A. LIÑÁN, Ignition and Extinction Fronts in Diffusion Flames, this volume.

[26] A.A. Liñán, Private Discussions, 1988/89.

[27] J.D. BUCKMASTER and M. MATALON, Anomalous Lewis Number Effects in Tribrachial Flames, 22nd Symp. (Int.) on Combustion, The Combustion Institute, 1988, pp. 1527-1535.

[28] W.T. Ashurst, Vorticity Generation in a Non-Premixed Flame Sheet, in 'Numerical Combustion,' A. Dervieux and B. Larrouturou (Eds.), Lecture Notes in Physics 351, Springer Verlag, 1989, pp. 3-21.

[29] Ya.B. Zeldovich, G.I. Barenblatt, V.B. Librovich and G.M. Makhviladze, 'The Mathematical Theory of Combustion and Explosions,' Consultants Bureau, New York, 1985. 